\title{
Validation of the Publication of New Names and New Combinations Previously Effectively Published Outside the IJSB
}

\author{
List No. $60 \dagger$
}

The purpose of this announcement is to effect the valid publication of the following new names and new combinations under the procedure described previously [Int. J. Syst. Bacteriol. 27(3):iv, 1977]. Authors and other individuals wishing to have new names and/or combinations included in future lists should send the pertinent reprint or a photocopy thereof to the IJSB (c/o ASM) for confirmation that all of the other requirements for valid publication have been met. It should be noted that the date of valid publication of these new names and combinations is the date of publication of this list, not the date of the original publication of the names and combinations. The authors of the new names and combinations are as given below, and these authors' names will be included in the author index of the present issue and in the volume author index in this issue of the IJSB.

Inclusion of a name on these lists validates the name and thereby makes it available in bacteriological nomenclature. The inclusion of a name on this list is not to be construed as taxonomic acceptance of the taxon to which the name is applied. Indeed, some of these names may, in time, be shown to be synonyms, or the organisms may be transferred to another genus, thus necessitating the creation of a new combination.

\begin{tabular}{|c|c|c|c|c|}
\hline Name & Proposed as: & Author(s) (reference) & Priority ${ }^{a}$ & Nomenclatural type $e^{b}$ \\
\hline Acetobacterium bakii & New species & Kotsyurbenko et al. (5) & 6 & Strain Z-4391 (= DSM 8239) \\
\hline Acetobacterium fimetarium & New species & Kotsyurbenko et al. (5) & 6 & Strain Z-4290 (= DSM 8238) \\
\hline Acetobacterium paludosum & New species & Kotsyurbenko et al. (5) & 6 & Strain Z-4092 (= DSM 8237) \\
\hline Arthrobacter cumminsii & New species & Funke et al. (3) & 4 & Strain DMMZ 445 (= DSM 10493) \\
\hline Arthrobacter woluwensis & New species & Funke et al. (3) & 4 & Strain CUL $1808(=$ DSM 10495) \\
\hline Clostridium fimetarium & New species & Kotsyurbenko et al. (4) & 6 & Strain Z-2189 (= DSM 9179) \\
\hline Desulfovibrio acrylicus & New species & van der Maarel et al. (7) & 5 & Strain W218 (= DSM 10141) \\
\hline Halomonas desiderata & New species & Berendes et al. (1) & 1 & Strain FB2 (= DSM 9502) \\
\hline Ruminococcus palustris & New species & Zhilina et al. (8) & 6 & Strain Z-7189 (= DSM 9172) \\
\hline Ruminococcus schinkii & New species & Rieu-Lesme et al. (6) & 3 & Strain B (= DSM 10518) \\
\hline Thiobacillus hydrothermalis & New species & Durand et al. (2) & 2 & Strain R3 (= DSM 7121) \\
\hline
\end{tabular}

$\dagger$ Lists 1 through 59 were published in the Int. J. Syst. Bacteriol. 27:306, 1977; 29:79, 436, 1979; 30:601, 676, 1980; 31:215, 382, 1981; 32:266, 384, 1982; 33:438, 672, 896,$1983 ; \mathbf{3 4}: 91$, 270, 355, 503, 1984; 35:223, 375, 535, 1985; 36:354, 489, 573, 1986; 37:179, 1987; 38:136, 220, 328, 449, 1988; 39:93, 205, 371, 495, 1989; 40:105, 212, $320,470,1990 ; 41: 178,331,456,580,1991 ; 42: 191,327,511,656,1992 ; 43: 188,398,624,864,1993 ; 44: 182,370,595,852,1994 ; 45: 197,418,619,879,1995 ;$ and 46:362, $625,836,1189,1996$.

${ }^{a}$ Priority number assigned according to the date the documentation and request for validation are received.

${ }^{b}$ Abbreviations: DSM, DSMZ-Deutsche Sammlung von Mikroorganismen und Zellkulturen GmbH, Braunschweig, Germany.

\section{REFERENCES}

1. Berendes, F., G. Gottschalk, E. Heine-Dobbernack, E. R. B. Moore, and B. J. Tindall. 1996. Halomonas desiderata sp. nov., a new alkaliphilic, halotolerant and denitrifying bacterium isolated from a municipal sewage works. Syst. Appl. Microbiol. 19:158-167.

2. Durand, P., A.-L. Reysenbach, D. Prieur, and N. Pace. 1993. Isolation and characterization of Thiobacillus hydrothermalis sp. nov., a mesophilic obligately chemolithotrophic bacterium isolated from a deep-sea hydrothermal vent in Fiji Basin. Arch. Microbiol. 159:39-44.

3. Funke, G., R. A. Hutson, K. A. Bernard, G. E. Pfyffer, G. Wauters, and M. D. Collins. 1996. Isolation of Arthrobacter spp. from clinical specimens and description of Arthrobacter cumminsii sp. nov. and Arthrobacter woluwensis sp. nov. J. Clin. Microbiol. 34:2356-2363.

4. Kotsyurbenko, O. R., A. N. Nozhevnikova, G. A. Osipov, N. A. Kostrikina, and A. M. Lysenko. 1995. A new psychroactive bacterium Clostridium fime- tarium, isolated from cattle manure digested at low temperature. Mikrobiologiya 64:804-810.

5. Kotsyurbenko, O. R., M. V. Simankova, A. N. Nozhevnikova, T. N. Zhilina, N. P. Bolotina, A. M. Lysenko, and G. A. Osipov. 1995. New species of psychrophilic acetogens: Acetobacterium bakii sp. nov., A. paludosum sp. nov., A. fimetarium sp. nov. Arch. Microbiol. 163:29-34.

6. Rieu-Lesme, F., B. Morvan, M. D. Collins, G. Fonty, and A. Willems. 1996. A new $\mathrm{H}_{2} / \mathrm{CO}_{2}$-using acetogenic bacterium from the rumen: description of Ruminococcus schinkii sp. nov. FEMS Microbiol. Lett. 140:281-286.

7. van der Maarel, M. J. E. C., S. van Bergeijk, A. F. van Werkhoven, A. M. Laverman, W. G. Meijer, W. T. Stam, and T. A. Hansen. 1996. Cleavage of dimethylsulfoniopropionate and reduction of acrylate by Desulfovibrio acrylicus sp. nov. Arch. Microbiol. 166:109-115.

8. Zhilina, T. N., O. R. Kotsyurbenko, G. A. Osipov, N. A. Kostrikina, and G. A. Zavarzin. 1995. Ruminococcus palustris sp. nov.-a psychroactive anaerobic organism from a swamp. Mikrobiologiya 64:674-680. 